\title{
30. DATA REPORT: ULTRAMAFIC REFERENCE MATERIAL FROM CORE 147-895D-10W ${ }^{1}$
}

\author{
H. Puchelt, ${ }^{2}$ J. Malpas, ${ }^{3}$ T. Falloon, ${ }^{4}$ R. Pedersen, ${ }^{5}$ J.-D. Eckhardt, ${ }^{2}$ and J.F. Allan ${ }^{6}$
}

\section{INTRODUCTION}

Ocean Drilling Program (ODP) Washcore 147-895D-10W is the last core taken in Hole 895D. It represents accidental recovery of drilling rubble during hole reaming, and therefore represents a mix of lithologies that could have been derived from 0 to 93.7 meters below seafloor (mbsf). It consists of 15 distinct pieces of serpentinized ultramafic and gabbroic rocks, with numerous gravel-sized pieces of mixed ultramafic material with small amounts of clay occurring at the bottom of the core. More uncurated rubble was sampled when trying to ream the hole. This uncurated rubble is not described in the Initial Reports volume, but was collected by one of the authors (H.P.). Petrographic inspection of the uncurated rubble showed it to be serpentinized olivine with margins of chrysotile asbestos, small amounts of spinels and gabbroic fragments. It was expected that the rubble composition represents well peridotites of the marine mantle, especially in relation to platinum group elements (PGE) (Prichard et al., this volume).

It was decided by the shipboard party to use this uncurated material as a reference for post-cruise geochemical analysis. On board, approximately $4 \mathrm{~kg}$ of the uncurated material (Sample 147-895D-10WReference 1) was washed clay-free with demineralized water, dried at $110^{\circ} \mathrm{C}$, and then ground in a motor-driven agate mortar. Because the grain size was not yet suitable after this procedure for preparing powder pellets aboard ship for X-ray fluorescence (XRF) trace-element analysis, three small splits were ground in tungsten carbide containers in a shatter box.

The results of shipboard XRF-analysis with an Applied Research Laboratories (ARL) machine gave preliminary trace elements only. The data are given in Table 1.

At the end of the cruise, small samples of approximately $200 \mathrm{~g}$ of 147-895D-10W-Reference 1 were distributed to interested cruise participants. H. Puchelt received additionally about $6 \mathrm{~kg}$ of uncurated rubble (Sample 147-895D-10W-Reference 2) for grinding and analysis at Karlsruhe. This material was carefully ground in portions of about $50 \mathrm{~g}$ in a shatter box with agate containers and was homogenized.

\section{ANALYTICAL TECHNIQUES}

On board JOIDES Resolution the following methods were used:

Wavelength dispersive XRF (WDXRF) for trace elements and gas chromatograph for $\mathrm{C}, \mathrm{S}, \mathrm{N}$, and $\mathrm{H}_{2} \mathrm{O}$ analysis.

The Bristol and Bergen groups used Inductively Coupled Plasma Mass Spectrometer (ICP-MS) (Kemp et al., unpubl. data) and

'Mével, C., Gillis, K.M., Allan, J.F., and Meyer, P.S. (Eds.), 1996. Proc. ODP, Sci. Results, 147: College Station, TX (Ocean Drilling Program).

${ }^{2}$ Institut für Petrographie und Geochemie der Universität Karlsruhe, Kaiserstrasse 12, D-76128 Karlsruhe, Federal Republic of Germany.

${ }^{3}$ Centre for Earth Resources Research, Memorial University, St. John's, Newfoundland AIB 3X5, Canada.

${ }^{4}$ Department of Geology, University of Bristol, Wills Memorial Building, Queen's Road, Bristol BS8 1RJ, United Kingdom.

${ }^{3}$ Geologisk Institutt, Universitet i Bergen, Allgaten 41, N.5007 Bergen, Norway

'Ocean Drilling Program, Texas A\&M University Research Park, 1000 Discovery Drive, College Station, TX 77845-9547, U.S.A.
WDXRF respectively. The Texas A\&M INAA techniques are given in Allan et al. (this volume).

In Karlsruhe the following methods were used:

1. energy dispersive XRF (EDXRF) (major and trace elements);

2. instrumental neutron activation (preferentially trace elements);

3. Karl Fischer titration (water determination);

4. ICP-MS (trace elements);

5. NiS fire-assay (platinum group elements, PGE);

6. infrared absorption for $\mathrm{CO}_{2}$ and $\mathrm{SO}_{2}$, carbon sulfur analyzer (CSA);

7. WDXRF (major and trace elements);

8. gas ion mass spectrometry for sulfur isotopes (Puchelt et al., this volume).

For methods 1-3 and 5-7 powders were used for WDXRF after fusion with lithiumtetraborate.

Method 4 required full disintegration with $\mathrm{HF}, \mathrm{HNO}_{3}$, and $\mathrm{HClO}_{4}$ in a closed Teflon container under pressure in a microwave oven.

\section{RESULTS}

The results of the Bristol, Bergen, Texas A\&M laboratories, and JOIDES Resolution for Sample 147-895D-10W-Reference 1 analysis are given in Table 1. The Karlsruhe group has analyzed the reference Sample 147-895D-10W-Reference 2 and analyzed Sample 147$895 \mathrm{D}-10 \mathrm{~W}-$ Reference 1 by INAA. When possible, replicate measurements were made.

Table 1 compares the results of the different groups and the two samples. Table 2 summarizes all the Karlsruhe results. Table 3 gives PGE values of 24 analyses by ICP-MS after fire-assay enrichment. The PGE data are presented in Figure 1. The normalized REE patterns of the reference are shown in Figure 2.

\section{CONCLUSIONS}

The REE data compare well between the Bristol, Texas, and Karlsruhe analyses for most elements in Sample 147-895D-10WReference 1. However, the major element analyses between Bergen and Karlsruhe show some differences between the two reference samples. Accuracy was determined in Bristol with international reference standards and in Karlsruhe by using single-element calibration curves and applying international reference standards (Kramar and Puchelt, 1982).

The PGE standard available for these analyses so far is a platinum ore (SARM 7). The PGE values of the ODP reference sample are in a range below $5 \mathrm{ppb}$, which makes it better suited as a standard for analysis of nonmineralized rocks.

Comparison of element data from Sample 147-895D-10W-Reference 1 and Sample 147-895D-10W-Reference 2 shows small differences in the values of some elements. We believe that the differences in element concentration are caused by nonhomogenous material. The two samples were not mixed before homogenization and analyses. We suggest that the remaining $4 \mathrm{~kg}$ of $147-895 \mathrm{D}-10 \mathrm{~W}-\mathrm{Refer}-$ ence 2, which is well homogenized and analyzed, be used as an ultra- 
mafic reference material. If more material is needed, the material from 147-895D-10W-Reference 1, stored in Texas, should be added to it and homogenized.

\section{REFERENCES}

Boynton, W.V., 1984. Cosmochemistry of the rare earth elements: meteorite studies. In Henderson, P. (Ed.), Rare Earth Element Geochemistry: New York (Elsevier), 63-114.
Kramar, U., and Puchelt, H., 1982. Reproducibility tests for INAA determinations with AGV-1, BCR-1 and GSP-1 and new data for 17 geochemical reference materials. Geostand. Newsl., 6:221-227.

Date of initial receipt: 11 August 1994

Date of acceptance: 14 June 1995

Ms 147SR-033

Table 1. Comparison of different working groups, methods, and samples for Samples 147-895D-10W-Reference 1 and 147-895D-10W-Reference 2 analysis.

\begin{tabular}{|c|c|c|c|c|c|c|}
\hline & \multirow{2}{*}{$\begin{array}{c}\text { 147-895D-10W } \\
\text { Reference } 2\end{array}$} & \multicolumn{5}{|c|}{$\begin{array}{c}147-895 \mathrm{D}-10 \mathrm{~W} \\
\text { Reference } 1\end{array}$} \\
\hline & & $\begin{array}{l}\text { Karlsruhe } \\
\text { INAA }\end{array}$ & $\begin{array}{l}\text { Bergen } \\
\text { WDXRF }\end{array}$ & $\begin{array}{l}\text { Texas } \\
\text { INAA }\end{array}$ & $\begin{array}{l}\text { Bristol } \\
\text { ICPMS }\end{array}$ & $\begin{array}{l}\text { Onboard } \\
\text { WDXRF }\end{array}$ \\
\hline \multicolumn{7}{|c|}{ Major elements (\%) } \\
\hline $\mathrm{SiO}_{2}$ & 39.20 & & 39.76 & & & \\
\hline $\mathrm{TiO}_{2}^{2}$ & 0.43 & & 0.386 & & & 0.34 \\
\hline $\mathrm{Al}_{2} \mathrm{O}_{3}$ & 3.56 & & 3.21 & & & \\
\hline $\mathrm{Fe}_{2} \mathrm{O}_{3}$ tot. & 9.34 & & 10.96 & 8.82 & & \\
\hline $\mathrm{MnO}$ & 0.15 & & 0.128 & & & \\
\hline $\mathrm{MgO}$ & 31.11 & & 31.358 & & & \\
\hline $\mathrm{CaO}$ & 3.27 & & 3.06 & & & \\
\hline $\mathrm{Na}_{2} \mathrm{O}$ & 0.44 & & 0.21 & 0.49 & & \\
\hline $\mathrm{K}_{2} \mathrm{O}$ & 0.05 & & 0.02 & & & \\
\hline $\mathrm{P}_{2} \mathrm{O}_{5}$ & 0.05 & & 0.04 & & & \\
\hline L.O.I. & & & 10.39 & & & \\
\hline $\mathrm{H}_{2} \mathrm{O}$ & 9.62 & & & & & \\
\hline $\mathrm{CO}_{2}$ & 0.95 & & & & & \\
\hline Total & 98.25 & & 99.522 & & & \\
\hline \multicolumn{7}{|c|}{ Minor elements (ppm) } \\
\hline $\mathrm{S}$ & 600 & & 1880 & & & \\
\hline $\mathrm{Cr}$ & & & 2290 & & & \\
\hline $\mathrm{Ni}$ & & & 1680 & & & \\
\hline $\mathrm{v}$ & 160 & & 55 & & & 94 \\
\hline $\mathrm{Cr}$ & 1826 & & 1976 & 2609 & & 2391 \\
\hline Co & 86 & & 25 & 85 & & 20 \\
\hline $\mathrm{Ni}$ & 1635 & & 1914 & 1553 & & 1766 \\
\hline $\mathrm{Cu}$ & 36 & & 19 & & & 20 \\
\hline $\mathrm{Zn}$ & 77 & & 69 & 72 & & 67 \\
\hline $\mathrm{Rb}$ & 1.3 & & 1 & $<5.2$ & & 0 \\
\hline $\mathrm{Sr}$ & 185 & & 231 & 213 & & 211 \\
\hline $\mathrm{Y}$ & 10 & & 10 & $<16.5$ & & 8 \\
\hline $\mathrm{Zr}$ & 28 & & 43 & & & 27 \\
\hline $\mathrm{Nb}$ & 0.92 & & 1 & & & 0 \\
\hline $\mathrm{Ba}$ & 10 & & 31 & & & 14 \\
\hline $\mathrm{La}$ & 127 & & 4 & & & \\
\hline $\mathrm{Ce}$ & 3.95 & & 0 & & & 2 \\
\hline Nd & 3.26 & & 0 & & & \\
\hline $\mathrm{Pb}$ & 10 & & 3 & & & \\
\hline Th & 0.09 & & 9 & & & \\
\hline $\mathrm{U}$ & 0.4 & & 2 & & & \\
\hline Mo & & & 2 & & & \\
\hline $\mathrm{La}$ & 1.27 & 1.00 & & 1.14 & 1.19 & \\
\hline $\mathrm{Ce}$ & 3.95 & 2.60 & & 3.08 & 3.16 & \\
\hline $\operatorname{Pr}$ & 0.6 & & & & 0.59 & \\
\hline $\mathrm{Nd}$ & 3.26 & & & & 2.64 & \\
\hline $\mathrm{Sm}$ & 1.1 & 0.90 & & 1.14 & 1.06 & \\
\hline Eu & 0.35 & 0.30 & & 0.32 & 0.34 & \\
\hline $\mathrm{Gd}$ & 1.52 & & & & 1.40 & \\
\hline $\mathrm{Tb}$ & 0.26 & 0.30 & & 0.23 & 0.24 & \\
\hline Dy & 1.74 & & & & 1.75 & \\
\hline Ho & 0.43 & 0.50 & & & 0.35 & \\
\hline Er & 1.06 & & & & 1.02 & \\
\hline $\mathrm{Tm}$ & 0.15 & & & & 0.12 & \\
\hline $\mathrm{Yb}$ & 1.02 & 0.90 & & 0.9 & 0.86 & \\
\hline $\mathrm{Lu}$ & 0.15 & 0.10 & & $<0.15$ & 0.15 & \\
\hline $\mathrm{Hf}$ & 0.8 & 0.6 & & 0.72 & & \\
\hline $\mathrm{Ta}$ & 0.05 & 0.06 & & $<0.04$ & & \\
\hline Th & 0.09 & $<0.1$ & & 0.13 & & \\
\hline $\mathrm{U}$ & 0.4 & $<0.07$ & & 0.49 & & \\
\hline
\end{tabular}

Notes: INAA = instrumental neutron activation analysis; WDXRF = wavelength dispersive $\mathrm{X}$-ray fluorescence. 
Table 2. Data resulting from Samples 147-895D-10W-Reference 2 analyzed in Karlsruhe by different methods.

\begin{tabular}{|c|c|c|c|c|c|c|c|c|c|c|c|c|c|}
\hline & \multicolumn{3}{|c|}{ WDXRF } & \multicolumn{3}{|c|}{ EDXRF } & \multicolumn{3}{|c|}{ ICP-MS } & \multicolumn{3}{|c|}{ INAA } & \multirow{2}{*}{$\begin{array}{l}\text { Recommended } \\
\text { values }\end{array}$} \\
\hline & Mean & SD & Repl. & Mean & SD & Repl. & Mean & SD & Repl. & Mean & SD & Repl. & \\
\hline \multicolumn{14}{|l|}{ Major elements (\%) } \\
\hline $\mathrm{SiO}_{2}$ & 39.20 & 0.043 & 4 & & & & & & & & & & 39.20 \\
\hline $\mathrm{TiO}_{2}^{2}$ & 0.43 & 0.002 & 4 & 0.44 & 0.04 & 9 & & & & & & & 0.43 \\
\hline $\mathrm{Al}_{2} \mathrm{O}_{3}$ & 3.56 & 0.007 & 4 & & & & & & & & & & 3.56 \\
\hline $\mathrm{Fe}_{2} \mathrm{O}_{3}$ tot. & 9.44 & 0.059 & 4 & 10.3 & 0.2 & 9 & & & & 9.23 & 0.19 & 9 & 9.34 \\
\hline $\mathrm{MnO}$ & 0.12 & 0.001 & 4 & 0.17 & 0.01 & 9 & & & & & & & 0.15 \\
\hline $\mathrm{MgO}$ & 31.11 & 0.017 & 4 & & & & & & & & & & 31.11 \\
\hline $\mathrm{CaO}$ & 3.27 & 0.010 & 4 & 3.56 & 0.11 & 9 & & & & & & & 3.27 \\
\hline $\mathrm{Na}_{2} \mathrm{O}$ & 0.44 & 0.002 & 4 & & & & & & & 0.56 & 0.04 & 9 & 0.44 \\
\hline $\mathrm{K}_{2} \mathrm{O}$ & 0.05 & 0.001 & 4 & 0.26 & 0.05 & 9 & & & & & & & 0.05 \\
\hline $\mathrm{P}_{2} \mathrm{O}_{5}$ & 0.05 & 0.001 & 4 & & & & & & & & & & 0.05 \\
\hline $\mathrm{H}_{2} \mathrm{O}$ & 9.62 & 0.159 & 5 & & & & & & & & & & 9.62 \\
\hline $\mathrm{CO}_{2}$ & 0.95 & 0.055 & 3 & & & & & & & & & & 0.95 \\
\hline Total & 98.25 & & & & & & & & & & & & 98.25 \\
\hline \multicolumn{14}{|c|}{ Minor elements (ppm) } \\
\hline S & 600 & 140 & 3 & & & & & & & & & & 600 \\
\hline $\mathrm{Li}$ & & & & & & & 2.7 & 0.11 & 5 & & & & 2.7 \\
\hline $\mathrm{Sc}$ & & & & & & & & & & 15 & 0.61 & 9 & 15 \\
\hline V & & & & & & & 160 & 5.27 & 5 & & & & 160 \\
\hline $\mathrm{Cr}$ & & & & & & & 1826 & 81.74 & 5 & 2497 & 325 & 9 & 1826 \\
\hline Co & & & & & & & 85 & 1.50 & 5 & 86 & 1.63 & 9 & 86 \\
\hline $\mathrm{Ni}$ & & & & & & & 1694 & 36.14 & 5 & 1575 & 57 & 9 & 1635 \\
\hline $\mathrm{Cu}$ & & & & 23 & 3.5 & 9 & 36 & 1.33 & 5 & & & & 36 \\
\hline $\mathrm{Zn}$ & & & & 66 & 10.0 & 9 & 77 & 4.32 & 4 & 88 & 30 & 9 & 77 \\
\hline $\mathrm{Ga}$ & & & & & & & 4.8 & 0.27 & 5 & & & & 4.8 \\
\hline As & & & & 6 & 0.9 & 9 & & & & & & & 6 \\
\hline $\mathrm{Br}$ & & & & & & & & & & 1.6 & 0.48 & 9 & 1.6 \\
\hline $\mathrm{Rb}$ & & & & & & & 1.3 & 0.07 & 5 & 6.8 & 1.48 & 9 & 1.3 \\
\hline $\mathrm{Sr}$ & & & & 189 & 16.3 & 9 & 179 & 23.81 & 5 & & & & 185 \\
\hline $\mathrm{Y}$ & & & & 10 & 1.0 & 9 & & & & & & & 10 \\
\hline $\mathrm{Zr}$ & & & & 31 & 1.8 & 9 & 25 & 2.54 & 5 & & & & 28 \\
\hline $\mathrm{Nb}$ & & & & & & & 0.92 & 0.06 & 5 & & & & 0.92 \\
\hline $\mathrm{Ag}$ & & & & & & & 0.93 & 0.21 & 5 & & & & 0.93 \\
\hline $\mathrm{Cd}$ & & & & & & & 0.74 & 0.16 & 5 & & & & 0.74 \\
\hline $\mathrm{Sb}$ & & & & & & & & & & 0.2 & 0.16 & 9 & 0.2 \\
\hline $\mathrm{Cs}$ & & & & & & & 0.59 & 0.57 & 4 & 0.15 & 0.04 & 9 & 0.15 \\
\hline $\mathrm{Ba}$ & & & & & & & 10 & 1.45 & 5 & & & & 10 \\
\hline $\mathrm{La}$ & & & & & & & 1.42 & 0.12 & 4 & 1.11 & 0.08 & 9 & 1.27 \\
\hline $\mathrm{Ce}$ & & & & & & & 4.01 & 0.20 & 4 & 3.88 & 0.89 & 9 & 3.95 \\
\hline $\mathrm{Pr}$ & & & & & & & 0.60 & 0.03 & 4 & & & & 0.6 \\
\hline Nd & & & & & & & 3.26 & 0.10 & 4 & 2.25 & 0.93 & 9 & 3.26 \\
\hline $\mathrm{Sm}$ & & & & & & & 1.10 & 0.02 & 4 & 1.10 & 0.08 & 9 & 1.1 \\
\hline Eu & & & & & & & 0.36 & 0.01 & 4 & 0.33 & 0.03 & 9 & 0.35 \\
\hline Gd & & & & & & & 1.52 & 0.04 & 4 & 1.52 & 0.71 & 9 & 1.52 \\
\hline $\mathrm{Tb}$ & & & & & & & 0.27 & 0.01 & 4 & 0.24 & 0.02 & 9 & 0.26 \\
\hline Dy & & & & & & & 1.74 & 0.04 & 4 & & & & 1.74 \\
\hline Ho & & & & & & & 0.37 & 0.01 & 4 & 0.48 & 0.10 & 9 & 0.43 \\
\hline Er & & & & & & & 1.06 & 0.02 & 4 & & & & 1.06 \\
\hline $\mathrm{Tm}$ & & & & & & & 0.16 & 0.00 & 4 & 0.13 & 0.04 & 9 & 0.15 \\
\hline $\mathrm{Yb}$ & & & & & & & 1.02 & 0.03 & 4 & 1.02 & 0.11 & 9 & 1.02 \\
\hline $\mathrm{Lu}$ & & & & & & & 0.16 & 0.00 & 4 & 0.14 & 0.02 & 9 & 0.15 \\
\hline Hf & & & & & & & 0.79 & 0.11 & 5 & 0.80 & 0.07 & 9 & 0.8 \\
\hline $\mathrm{Ta}$ & & & & & & & $<0.05$ & & 4 & 0.05 & 0.01 & 9 & 0.05 \\
\hline $\mathrm{Tl}$ & & & & & & & $<0.1$ & & 5 & & & & $<0.1$ \\
\hline $\mathrm{Pb}$ & & & & 10 & 1.8 & 9 & 6.8 & 4.65 & 5 & & & & 10 \\
\hline $\mathrm{Bi}$ & & & & & & & $<0.1$ & & 5 & & & & $<0.1$ \\
\hline $\mathrm{Th}$ & & & & & & & 0.08 & 0.01 & 4 & 0.09 & 0.01 & 9 & 0.09 \\
\hline $\mathrm{U}$ & & & & & & & 0.35 & 0.05 & 5 & 0.45 & 0.04 & 9 & 0.4 \\
\hline \multicolumn{14}{|l|}{ Trace elements (ppb) } \\
\hline $\mathrm{Ru}$ & & & & & & & 4.3 & 1.0 & 24 & & & & 4.3 \\
\hline $\mathrm{Pd}$ & & & & & & & 4.3 & 1.0 & 24 & & & & 4.3 \\
\hline Ir & & & & & & & 1.6 & 0.4 & 24 & & & & 1.6 \\
\hline $\mathrm{Pt}$ & & & & & & & 4.0 & 1.3 & 24 & & & & 4.0 \\
\hline $\begin{array}{l}\text { S-Isotopes AVS } \\
\delta^{34} \text { S Pyrite }\end{array}$ & (\%o) & $\begin{array}{c}-10.0 \\
0\end{array}$ & & & & & & & & & & & \\
\hline
\end{tabular}

Note: WDXRF $=$ wavelength dispersive $\mathrm{X}$-ray fluoresence; $\mathrm{EDXRF}=$ energy dispersive $\mathrm{X}$-ray fluoresence; $\mathrm{ICP}-\mathrm{MS}=$ trace elements; INAA = instrumental neutron activation analysis; $\mathrm{SD}=$ standard deviation, Repl. $=$ replicates . 
DATA REPORT

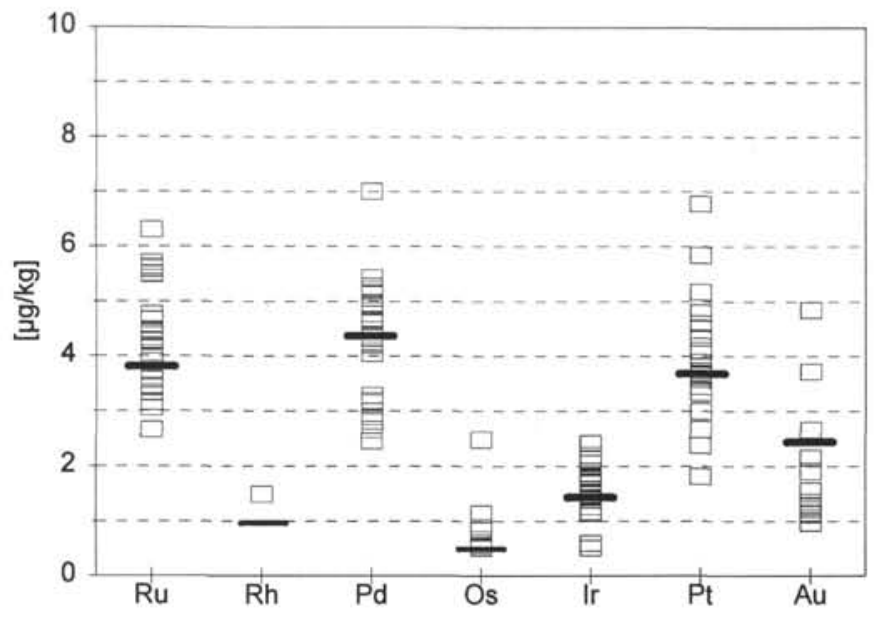

Figure 1. PGE data in pps of 147-895D-10W-Reference $2(n=24)$. Heavy lines are mean values for Ru (3.8 pps), Pd (4.3 pps), Ir (1.4 pps), Pt (3.7 $\mathrm{ppb})$, and $\mathrm{Au}(2.4 \mathrm{ppb})$ and lighter lines are detection limits for $\mathrm{Rh}(1 \mathrm{ppb})$, and $\mathrm{Os}(0.5 \mathrm{ppb})$.

Table 3. PGE values for 147-895D-10W-Reference 2 from 24 replicates analyzed in Karlsruhe.

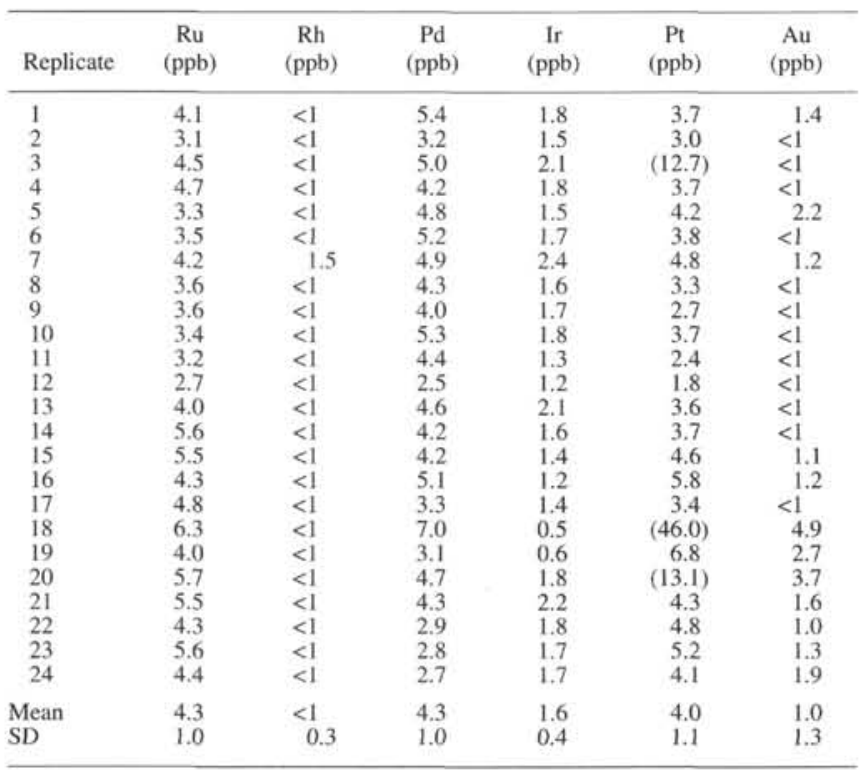

Note: $\mathrm{SD}=$ standard deviation; values in brackets are not included in mean value.

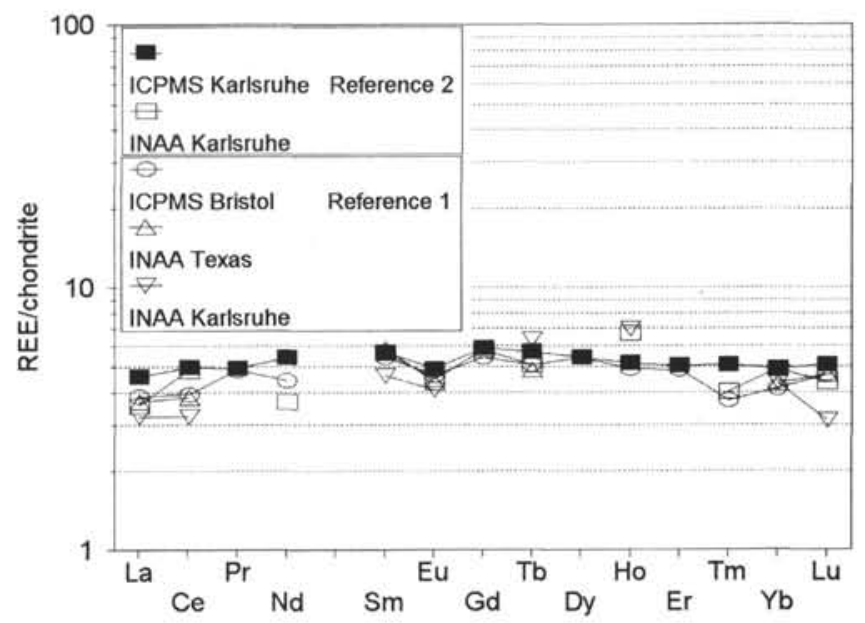

Figure 2. Comparison of the normalized REE patterns of the two samples and of the different working groups and methods. REE are normalized to Boynton chondrite (Boynton, 1984, table 3.3)

496 